Pacific Journal of Mathematic 


\title{
AN OBSTRUCTION TO LIFTING CYCLIC MODULES
}

\author{
MELVIN HOCHSTER
}

Let $A$ be a ring (all ring are commutative, with identity), let $t \in A$ be a nonzerodivisor and not a unit, and let $B=A / A t$. Let $M$ be a $B$-module of finite type. We call an $A$-module $E$ of finite type a lifting of $M$ (or we say that " $E$ lifts $M$ " or " $M$ lifts to $E$ ") if (1) $t$ is not a zerodivisor on $E$ and (2) $E / t E \cong M$. Grothendieck's lifting problem (GLP) is this: Suppose that $(A, m)$ is a complete regular local ring and that $t \in m-m^{2}$, so that $B=A / t A$ is again regular. If $M$ is a $B$-module of finite type, does $M$ lift to $A$ ? $\mathrm{A}$ simple and completely elementary counterexample is given below for the case where $M$ is cyclic.

One of the motivations for studying GLP is the possibility of settling Serre's conjecture [9] p. V-14 on multiplicities using lifting: this idea is discussed in [1], [2], and [3], where affirmative answers to the question are obtained in some cases. The terminology "Grothendieck's lifting problem" is taken from these papers. The first published discussion of the relevance of the lifting problem to multiplicities of which I am aware is in Nastold's paper [6], which was brought to my attention by the referee. (Of course, if $A$ is equicharacteristic complete regular and $t \in m-m^{2}$ one can always lift, for then $A \cong$ $B[[t]]$.

In the general case, it seems that one can construct counterexamples by completing the example [8] of Serre of an unliftable variety along lines suggested by Laudal and Kleppe, but the example in [8] is complicated, hard to write down, and, so far as I know, the details of the proof that one can complete have not appeared.

In this note we give a different counterexample to lifting even cyclic modules in the context of GLP. (Later, we also propose a weaker lifting conjecture which would suffice for the multiplicities problem and to which I know no counterexamples.) The method for constructing counterexamples is totally elementary, and provides examples when $B$ is a complete regular local ring of mixed characteristic as well as when $B$ has positive characteristic. The obstruction to lifting we use is quite coarse: it comes out of the all but trivial (and, I assume, well known) lemma below. Much of the credit for focusing attention on this obstruction belongs to D. Ferrand.

Before stating the lemma we make some special conventions for the cyclic case. Suppose that $(A, m)$ is a local ring and we wish to lift $M=A / J$, where $t \in J \varsubsetneqq A$, a typical cyclic $B$-module, to $A$. By 
Nakayama's lemma the lifting $E$ must be a cyclic $A$-module $A / I$, and it is evident that the problem of lifting $J$ is equivalent to the problem of finding an ideal $I \subset J$ such that $J=I+A t$ and $t$ is not a zerodivisor mod. I. Thus, if $A$ is any ring, $t$ a nonzerodivisor, $t \in J \varsubsetneqq A$, we call $I$ a $t$-lifting (or, simply, a lifting) of $J$, etc. if $J=I+A t$ and $t$ is not a zerodivisor mod. $I$.

Let $C=A / J$ and let $x$ be an indeterminate. We shall denote by $\phi$ the map of graded $C$-algebras $C[x] \rightarrow g r_{J} A$ which maps $x$ to the class $t+J^{2}$ of $t$ in $J / J^{2}$.

LemMa. Let $A$ be a ring, $t$ a nonzerodivisor, and suppose $t \in$ $J \varsubsetneqq A$. Let $C=A / J$ and $\phi$ be as above. If $J$ has a t-lifting then there is a map $\psi: g r_{J} A \rightarrow C[x]$ of graded $C$-algebras such that $\psi \phi=\mathrm{id}_{C[x]}$. In particular:

(1) $\phi$ is injective.

(2) For each $n$ the map of $C$-modules $C \rightarrow J^{n} / J^{n+1}$ which takes the class of 1 to the class of $t^{n}$ embeds $C$ in $J^{n} / J^{n+1}$ as a direct summand.

(3) For every positive integer $n, J^{n+1}: A t^{n}=J$.

CoROLlaRY. With the hypothesis of the Lemma, if $J$ has a $t$-lifting then the map $h: C=A / J \rightarrow J / J^{2}$ which takes $1+J$ to $t+J^{2}$ embeds $A / J$ as a direct summand of $J / J^{2}$. In particular, it is injective, and, hence, $J^{2}: A t=J$.

Proof. To construct $\psi$ we give explicitly its $n^{\text {th }}$ graded piece $\psi_{n}: J^{n} / J^{n+1} \rightarrow A / J$ (we identity $A / J$ with $(A / J) x^{n}$, by sending $1 \mapsto x^{n}$ ). We want $\psi_{n}\left(t^{n}+J^{n+1}\right)=1+J$. Now $J^{n}=(I+A t)^{n} \subset I+A t^{n}$. We have a composite map: $J^{n} \longleftrightarrow \mathrm{I}+A t^{n} \rightarrow\left(I+A t^{n}\right) / I \cong(A / I) t^{n} \stackrel{\alpha}{\cong} A / I \rightarrow$ $A / J \cong(A / J) x^{n}$ where the maps are the obvious inclusions, quotient maps, and identifications, except for $\alpha: \alpha$ is the inverse of the map $A / I \rightarrow(A / I) t^{n}$ which sends $1 \mapsto t^{n}$, which is an isomorphism precisely because $t$ is not a zerodivisor modulo $I$. This $\alpha\left(t^{n}\right)=1$, and the composite map $J^{n} \rightarrow A / J$ sends $t^{n}$ to $1+J$. We let $\psi_{n}$ be the induced map $J^{n} / J^{n+1} \rightarrow A / J \cong(A / J) x^{n}$. It is clear that $\psi_{n}$ is a $C$-module retraction. The $\psi_{n}$ fit together to give a homomorphism of graded algebras, for if $u \in J^{m}, v \in J^{n}, u+J^{m+1}$ maps to $a+J$ in $A / J$, and $v \in J^{n+1}$ maps to $b+J$ in $A / J$, then $u v \in J^{m+n}$ represents an element of $J^{m+n} / J^{m+n+1}$; moreover, if $u=i_{1}+\left(a+j_{1}\right) t^{m}$ and $v=i_{2}+\left(b+j_{2}\right) t^{n}$, where $i_{1}, i_{2} \in$ $I, j_{1}, j_{2} \in J$ then $u v=i_{3}+\left(a b+j_{3}\right) t^{m+n}$, clearly, where $i_{3} \in I, j_{3} \in J$, whence $\psi_{m+n}\left(u v+J^{m+n+1}\right)=\psi_{m}\left(u+J^{m+1}\right) \psi_{n}\left(v+J^{n+1}\right)$.

Now, (1) and (2) are clear, while (3) is readily seen to be a restatement of the fact that $\phi_{n}$ is injective. The Corollary is just the special case $n=1$. 
EXAMPLE 1. We now observe that even the condition $J^{2}: A t=J$ obstructs lifting in the context of GLP. Let $V$ be a complete discrete valuation ring with maximal ideal generated by 2 , let $K=V / 2 V$, let $x_{i j}, 1 \leqq i \leqq 3,1 \leqq j \leqq 2$ be six analytic indeterminates over $V$, let $A=V\left[\left[X_{i, j}\right]\right]_{i, j}$, let $t=2$, let $q=\sum_{i=1}^{3} x_{i 1} x_{i 2}$ and let $J=\left(2, q,\left\{x_{i, j}^{2}\right\}\right)_{i, j} A$. Then $J$ has no $t$-lifting $I$ : in fact $J^{2}: 2 A \not \subset J$. To see this let $D=$ $\sum_{i=1}^{3} x_{i 1} x_{i 2} x_{i+1,1} x_{i+1,2}$ (where the first subscript is taken mod. 3). Then $2 D=q^{2}-\sum_{i=1}^{3}\left(x_{i 1}^{2}\right)\left(x_{i 2}^{2}\right) \in J^{2}$ and $D \in J^{2}: 2 A$, but $D \notin J$. To prove that $D \notin J$ we work mod. $H=\left(2,\left\{x_{i, j}^{2}\right\}_{i, j}\right) A$. Let ' denote residues mod. $H$. $A / H$ is a graded finite dimensional $K$-algebra and any degree four element of $J(A / H)=q^{\prime}(A / H)$ is in the $K$-span of the products $q^{\prime} \cdot\left(E^{\prime} \cup F^{\prime}\right)$ where $E=\left\{x_{i 1} x_{i 2}: 1 \leqq i \leqq 3\right\}$ and $F=\left\{x_{i j} x_{i^{*} j *}: 1 \leqq i<i^{*} \leqq\right.$ $\left.3,1 \leqq j \leqq 2,1 \leqq j^{*} \leqq 2\right\}$. ( $E^{\prime} \cup F^{\prime}$ gives all square-free terms.) Each element of $q^{\prime} \cdot E^{\prime}$ is the sum of two of the three monomials in $D^{\prime}$ while if $u=x_{i j} x_{i^{*} j^{*}} \in F$, then, mod. $H, u \equiv x_{i j} x_{i^{*} j *} x_{i^{\prime \prime 1} 1} x_{i^{\prime \prime} 2^{\prime}}$ where $i^{\prime \prime}$ is the unique element of $\{1,2,3\}-\left\{i, i^{*}\right\}$, and these twelve distinct monomials do not occur in $D^{\prime}$ at all. Thus, $D^{\prime}$ is not in the $K$-span of $q^{\prime} \cdot\left(E^{\prime} \cup E^{\prime}\right)$ and $D \notin J$.

ExAmple 2. Let $V, K, A, q, J$ and $D$ be precisely the same as in Example 1, and let $u \in J^{2}$. Let $t_{u}=2+u$. Then $D t_{u}=2 D+$ $u D \in J^{2}$, and $D \notin J$. Thus, we have $J^{2}: A t_{u} \not \subset J$ again, but now $B=$ $A / A t_{u}$ is a regular local ring of mixed characteristic (rather than characteristic 2) if we choose $u$ properly, e.g. $u=x_{11}^{4}+x_{12}^{2}+x_{11}^{2}$, and the ramification of 2 cannot be absorbed into the coefficient ring of $B$, i.e. $B$ is precisely the kind of regular local ring for which Serre's conjecture is not known.

REMARK 1. Similar examples undoubtedly exist for every positive prime characteristic $p$.

REMARK 2. One can consider more general lifting problems, which were open for a while. Let $A \rightarrow B$ be a homomorphism, and let $M$ be a $B$-module of finite type. Call an $A$-module $E$ of finite type a lifting of $M$ if (1) $\operatorname{Tor}_{i}^{A}(B, E)=0$ if $i \geqq 1$ and (2) $B \otimes_{A} E \cong M$. Suppose that $B=A / A t$, where $t$ is a nonzerodivisor. Then condition 1 ) is equivalent to the assertion that $t$ is not a zerodivisor on $B$. One can ask, if $p d_{B} M<\infty$, does $M$ lift to $A$ ? The technique of Peskine-Szpiro [7], Ch. I, $\S 2$ gives counterexamples even if $(A, m)$ is a complete regular local ring of equicharacteristic $0, B=A / A t$, and $p d_{B} M=2$. However, in their examples $t \in m^{2}$ and $B$ is not regular: in fact, their obstruction is that a certain $B$-module has infinite projective dimension when it should have finite projective dimension, and so cannot provide examples with $B$ regular. 
REMARK 3. The obstruction indicated in the Corollary, i.e. that the map $h: A / J \rightarrow J / J^{2}$ which takes $1+J$ to $t+J^{2}$ split, is precisely the obstruction to lifting $A / J$ "as far as" $A / t^{2} A$. For if $s: J / J^{2} \rightarrow A / J$ is a splitting (left inverse for $h$ ) then if $I_{1}=\operatorname{Ker}\left(J / J^{2} \stackrel{s}{\rightarrow}\right.$ $A / J), A / I_{1}$ "lifts" $A / J$ "as far as" $A / t^{2} A$. If one has a good criterion for lifting from "mod. $t$ " to "mod. $t$ ", then one can iterate it to lift, successively, mod. $t^{4}$, mod. $t^{8}$, etc., and, utlimately, given $t$-adic completeness, back to the original ring. This idea motivated some of the more general lifting conjectures.

REMARK 4. It is clear that in some sense the problem with lifting from $B$ to $A$ in the context of GLP is $p$-torsion: in the examples here, even when $p \neq 0$ in the regular ring $B=A / A t$, there is $p$-torsion on $A / J$. I believe it would be too naive to conjecture that simply because there is no $p$-torsion on $A / J$ that one can lift; on the other hand, I feel there is almost certainly a good result along the following lines: "If there is no $p$-torsion on the following modules $\cdots$ (canonically associated with $J, t$ ), then $J$ lifts".

For example, we have the following:

Proposition. Let $(A, m)$ be a regular local ring of mixed characteristic, let $t \in m-m^{2}$, suppose $t \in J \varsubsetneqq A$, and let $C=A / J$. Let $p=$ $\operatorname{char}(A / m)$. If $p$ is not a zerodivisor on $C$ and also not a zerodivisor on $\operatorname{Ext}_{C}^{1}\left(J /\left(A t+J^{2}\right), C\right)=E$, then the map $h: C \rightarrow J / J^{2}$ which takes $1+J$ to $t+J^{2}$ splits.

Proof. If $h$ does not split them either (i) $h$ is not injective or (ii) $h$ is injective and the exact sequence

$$
0 \longrightarrow C \stackrel{h}{\longrightarrow} J / J^{2} \longrightarrow J /\left(A t+J^{2}\right) \longrightarrow 0
$$

is not split, i.e. represents a nonzero element of $E=\operatorname{Ext}_{C}^{1}\left(J /\left(A t+J^{2}\right), C\right)$. Since $p$ is not a zerodivisor on $C, E$, both situations (i) and (ii) are preserved upon localizing at $p$ : hence there is a prime $Q \supset J$ such that $p \notin Q$ and the splitting fails after localizing at $Q$ (or, equivalently, at $Q / J)$. But then if we localize at $Q$ and complete, the splitting will still fail. This is impossible: $\hat{A}_{Q}$ is complete and equicharacteristic 0 , so that $J \hat{A}_{Q}$ does have a $t$-lifting.

For the rest of this note we restrict our attention to the following situation:

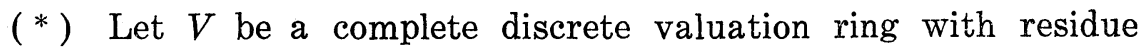
class field $K$ of characteristic $p>0$, and suppose $0 \neq p$ in $V$ generates the maximal ideal. Let $A$ be $V\left[\left[x_{1}, \cdots, x_{n}\right]\right]$ and let $t$ be a regular 
parameter in $A$ such that $p \neq 0$ in the regular local ring $B=A / A t$. Let $t \in J \varsubsetneqq A$; and suppose that $J$ is prime.

We want to make some observations about this situation.

Observation 1. In order to prove Serre's conjecture on multiplicities it would suffice to prove that $J$ has a $t$-lifting in this situation. To emphasize that $J$ is prime, we write " $P$ " instead of " $J$ " i.e. $P=J$.

Observation 2. Under the hypothesis $\left(^{*}\right) h: A / J \rightarrow J / J^{2}$ (or $A / P \rightarrow$ $\left.P / P^{2}\right)$ is automatically injective, i.e. $p^{2}: A t=P$. For $t$ is a regular parameter $\Rightarrow A / t A$ is regular $\Rightarrow(A / t A)_{P / t A} \cong A_{P} / t A_{P}$ is regular $\Rightarrow t$ is a regular parameter in the regular local ring $A_{P} \Rightarrow P^{2} A_{P}: t A_{P}=P A_{P}$ and $P^{2}: A t \subset\left(P^{2} A_{P}: t A_{P}\right) \cap A=P A_{P} \cap A=P$. However it is completely unclear why there should be any reason for $h$ to split.

Observation 3. Under the hypothesis $\left(^{*}\right)$, if $h: A / P \rightarrow P / P^{2}$ splits, then the map $\phi:(A / P)[x] \rightarrow g r_{P} A$ of graded $C$-algebras (where $C=A / P$ ) splits. For if $s$ splits (is a left inverse for) $h, s$ induces a graded $C$-algebra map $\varepsilon: \operatorname{symm}_{C} P / P^{2} \rightarrow \operatorname{symm}_{C} C \cong C[x]$. If we localize at $P$, since $A_{P}$ is regular we have an isomorphism: $\operatorname{symm}_{C}\left(P / P^{2}\right)_{P} \stackrel{\beta}{\rightarrow}$ $g r_{P C_{P}} A_{P}$ (both are polynomial rings in height $P$ variables over the field $C_{P}=(A / P)_{P^{\prime}}$ and the map is induced by an isomorphism of their first graded pieces). Now, $\beta$ is induced by localization from the natural map $\delta: \operatorname{symm}_{C}\left(P / P^{2}\right) \rightarrow g r_{P} A$ (which in turn is induced from the inclusion of $P / P^{2}$ as the one-forms of $\left.g r_{P} A\right)$. Hence, we have a commutative diagram:

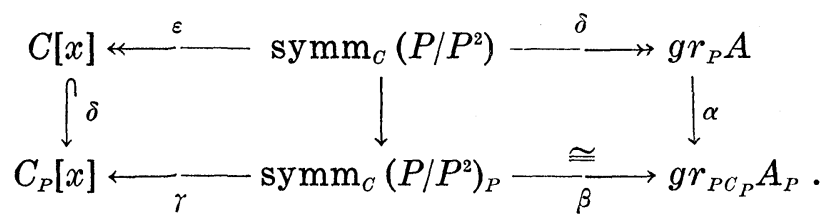

Since $\beta$ is an isomorphism, we have a homomorphism $\psi=\gamma \beta^{-1} \alpha: g r_{P} A \rightarrow$ $C_{P}[x]$. Since $\delta$ is surjective, $\operatorname{Im} \psi=\operatorname{Im} \psi \delta=\operatorname{Im} \delta \varepsilon=C[x] \subset C_{P}[x]$. Thus, $\psi$, with its range restricted, is the (unique) graded $C$-algebra homomorphism which makes the diagram

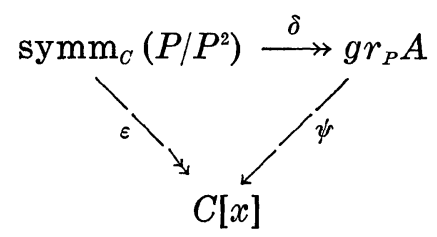

commute, and it follows from the definition of $\varepsilon$ that $\psi$ is a left 
inverse for $\phi$.

This shows that under the hypothesis (*), the "main case", the entire obstruction to lifting presented by the Lemma is no worse than the obstruction presented by the Corollary. The graded algebra map splits if and only if $h$ splits.

However, it appears that even if the graded algebra map splits, we have merely taken a feeble first step towards lifting $J$.

Observation 4. It is useful to put the obstruction to splitting given by the Corollary in a more concrete form. We therefore note:

Proposition. Under the hypothesis (*) (so that $J=P$ is prime), the $\operatorname{map} h: C=A / J \rightarrow J / J^{2}$ splits if and only if for every (irreducible) $A$-ideal $L \supset J$ (primary to the maximal ideal $m$ of $A$ ),

$$
L J: A t=L \text {. }
$$

Either or both of the parenthetical phrases may be omitted without affecting the validity of this statement.

In fact, $A / J \rightarrow J / J^{2}$ makes $A / J$ a direct summand if and only if $A / J$ is a pure submodule, and since $A / J$ is a complete local domain, this holds if and only if for every ideal $L / J$ of $A / J, h^{-1}\left(L\left(J / J^{2}\right)\right)=$ $(A / J)$ (i.e. $A / J \rightarrow J / J^{2}$ is cyclically pure: see [4]), and it suffices to know this for $(m / J)$-primary irreducible ideals $L / J$ (again, see [4]). But $h^{-1}\left(L\left(J / J^{2}\right)\right)=L(A / J)$ if and only if $L J: A t=L$.

We conclude with the following point: Serre's conjecture on multiplicities over a regular local ring $B=A / A t$ is known except in the situation of $\left(^{*}\right)$, and we may further suppose that $t$ is an Eisenstein polynomial. To deduce the result for $B$ from the result for $A$, it would suffice to know the following:

Weak lifting conjecture. Under the hypothesis $\left({ }^{*}\right)$, let $Q$ be a prime of $B$ such that $p \notin Q$. Then some B-module $M$ whose support is defined by $Q$ lifts to $A$.

Proof that the weak lifting conjecture implies Serre's conjecture. It suffices to show (cf. [8] Ch. V, §4) that for each pair $\left(Q, Q_{1}\right)$ of primes of $B$ with $Q+Q_{1}$ of coheight $0, e_{B}\left(B / Q, B / Q_{1}\right) \geqq 0$, with inequality $\Leftrightarrow \operatorname{dim}(B / Q)+\operatorname{dim}\left(B / Q_{1}\right)=\operatorname{dim} B . \quad\left(\right.$ Here $e_{B}(M, N)=$ $\sum_{i=0}^{\operatorname{dim} B}(-1)^{i} l\left(\operatorname{Tor}_{i}^{B}(M, N)\right.$.) Choose a counterexample with $\operatorname{dim}(B / Q)+$ $\operatorname{dim}\left(B / Q_{1}\right)$ as small as possible. If $p \in Q$ and $p \in Q_{1}$, then the result is known ([5], Prop. 4). Now suppose, say, $p \notin Q$. Choose $M$ such 
that Supp $M=\{P \in \operatorname{Spec}(B): P \supset Q\}$ and $M$ has a lifting $E$ to $A$. Then $\operatorname{dim} A-\operatorname{dim} E-\operatorname{dim}\left(B / Q_{1}\right)=\operatorname{dim} B-\operatorname{dim} M-\operatorname{dim}\left(B / Q_{1}\right)$ and $\operatorname{dim} M=\operatorname{dim}(B / Q)$. Thus $e_{A}\left(E, B / Q_{1}\right) \geqq 0$ with inequality if and only if $\operatorname{dim}(B / Q)+\operatorname{dim}\left(B / Q_{1}\right)=\operatorname{dim} B . \quad$ Each $\operatorname{Tor}_{i}^{A}\left(E, B / Q_{1}\right)$ is isomorphic with $\operatorname{Tor}_{i}^{B}\left(M, B / Q_{1}\right)$, and $M$ has a prime filtration involving a positive number $\lambda$ of copies of $B / Q$ and certain other primes $Q_{j}^{*}$ such that $Q_{j}^{*} \supsetneq Q$. Hence $e_{A}\left(E, B / Q_{1}\right)=e_{B}\left(M, B / Q_{1}\right)=\left(\right.$ for suitable $\left.\mu_{j}\right) \lambda e_{B}(B / Q$, $\left.B / Q_{1}\right)+\sum_{j} \mu_{j} e_{B}\left(B / Q_{j}^{*}, B / Q_{1}\right)=\lambda e_{B}\left(B / Q, B / Q_{1}\right)$ because $\operatorname{dim}\left(B / Q_{j}^{*}\right)+$ $\operatorname{dim}\left(B / Q_{1}\right)<\operatorname{dim}(B / Q)+\operatorname{dim}\left(B / Q_{1}\right)$ (so that the result holds for $\left(Q_{j}^{*}, Q_{1}\right)$, and $\operatorname{dim}\left(B / Q_{j}^{*}\right)+\operatorname{dim}\left(B / Q_{1}\right)<\operatorname{dim} B \Rightarrow e_{B}\left(B / Q_{j}^{*}, B / Q_{j}\right)=0$ for each $j)$. Thus $e_{B}\left(B / Q, B / Q_{1}\right)=(1 / \lambda) e_{A}\left(E, B / Q_{1}\right) \geqq 0$, with inequality if and only if $\operatorname{dim}(B / Q)+\operatorname{dim}\left(B / Q_{1}\right)=\operatorname{dim} B$.

I know no counterexamples to this weak lifting conjecture.

\section{REFERENCES}

1. D. A. Buchsbaum and D. Eisenbud, Lifting modules and a theorem on finite free resolutions, Ring Theory, Academic Press, New York, (1972), 63-74.

2. - Remarks on ideals and resolutions, Symposia Math., XI (1973), 193-204.

3. - Some structure theorems for finite free resolutions, Advances in Math., 12 (1974), 84-139.

4. M. Hochster, Cyclic purity versus purity in excellent Noetherian rings, preprint.

5. $M^{\mathrm{me}}$ M.-P. Malliavin-Brameret, Structure d'anneaux réguliers et caractéristiques d'Euler-Poincaré, Bull. Sc. Math. $2^{\mathrm{e}}$ série, 97 (1973), 81-88.

6. H.-J. Nastold, Zur Serreschen Multiplizitätstheorie in der arithmetischen Geometrie, Math. Annalen, 143 (1961), 333-343.

7. C. Peskine and L. Szpiro, Dimension projective finite et cohomologie locale, Publ. I. H. E.S. Paris, No. 42, (1973), 323-395.

8. J.-P. Serre, Exemples de variétés projectives en caractéristique $p$ non relèvables en caractéristique zéro, Proc. Nat. Acad. Sci., 47 (1961), 108-109.

9. - Algèbre locale. Multiplicités, Springer-Verlag Lecture Notes in Math. No. 11, New York, 1965.

Received May 13, 1975. Research supported in part by a grant from the National Science Foundation.

PuRdue UNIVERSity 



\section{PACIFIC JOURNAL OF MATHEMATICS}

\section{EDITORS}

RICHARD ARENS (Managing Editor)

University of California

Los Angeles, California 90024

\author{
R. A. Beaumont \\ University of Washington \\ Seattle, Washington 98105
}

\section{J. DugundjI}

Department of Mathematics University of Southern California Los Angeles, California 90007

D. Gilbarg and J. Milgram

Stanford University

Stanford, California 94305

\section{ASSOCIATE EDITORS}
E. F. BECKENBACH
B. H. NeumanN
F. WOLF
K. YoshidA

\section{SUPPORTING INSTITUTIONS}

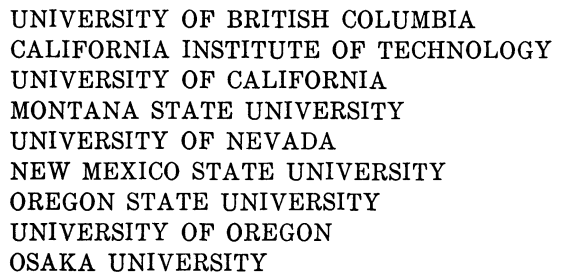

UNIVERSITY OF BRITISH COLUMBIA CALIFORNIA INSTITUTE OF TECHNOLOGY UNIVERSITY OF CALIFORNIA MONTANA STATE UNIVERSITY UNIVERSITY OF NEVADA NEW MEXICO STATE UNIVERSITY OSAKA UNIVERSITY OREGON STATE UNIVERSITY UNIVERSITY OF OREGON

\author{
UNIVERSITY OF SOUTHERN CALIFORNIA \\ STANFORD UNIVERSITY \\ UNIVERSITY OF TOKYO \\ UNIVERSITY OF UTAH \\ WASHINGTON STATE UNIVERSITY \\ UNIVERSITY OF WASHINGTON \\ AMERICAN MATHEMATICAL SOCIETY
}

The Supporting Institutions listed above contribute to the cost of publication of this Journal, but they are not owners or publishers and have no responsibility for its content or policies.

Mathematical papers intended for publication in the Pacific Journal of Mathematics should be in typed form or offset-reproduced, (not dittoed), double spaced with large margins. Please do not use built up fractions in the text of your manuscript. You may however, use them in the displayed equations. Underline Greek letters in red, German in green, and script in blue. The first paragraph or two must be capable of being used separately as a synopsis of the entire paper. Items of the bibliography should not be cited there unless absolutely necessary, in which case they must be identified by author and Journal, rather than by item number. Manuscripts, in triplicate, may be sent to any one of the editors. Please classify according to the scheme of Math. Reviews, Index to Vol. 39. All other communications should be addressed to the managing editor, or Elaine Barth, University of California, Los Angeles, California, 90024.

The Pacific Journal of Mathematics expects the author's institution to pay page charges, and reserves the right to delay publication for nonpayment of charges in case of financial emergency.

100 reprints are provided free for each article, only if page charges have been substantially paid. Additional copies may be obtained at cost in multiples of 50 .

The Pacific Journal of Mathematics is issued monthly as of January 1966. Regular subscription rate: $\$ 72.00$ a year $(6$ Vols., 12 issues). Special rate: $\$ 36.00$ a year to individual members of supporting institutions.

Subscriptions, orders for back numbers, and changes of address should be sent to Pacific Journal of Mathematics, 103 Highland Boulevard, Berkeley, California, 94708.

PUBLISHED BY PACIFIC JOURNAL OF MATHEMATICS, A NON-PROFIT CORPORATION

Printed at Kokusai Bunken Insatsusha (International Academic Printing Co., Ltd.), 8-8, 3-chome, Takadanobaba, Shinjuku-ku, Tokyo 160, Japan. 


\section{Pacific Journal of Mathematics}

\section{Vol. 61, No. 2 December, 1975}

Graham Donald Allen, Francis Joseph Narcowich and James Patrick Williams, An operator version of a theorem of Kolmogorov .......................

Joel Hilary Anderson and Ciprian Foias, Properties which normal operators share with normal derivations and related operators . . . . . . . . . . . . . . . . . . . . .

Constantin Gelu Apostol and Norberto Salinas, Nilpotent approximations and

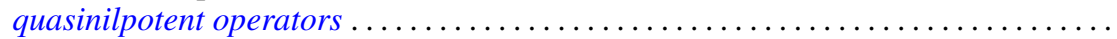

James M. Briggs, Jr., Finitely generated ideals in regular $F$-algebras . . . . . . . . . . .

Frank Benjamin Cannonito and Ronald Wallace Gatterdam, The word problem and power problem in 1-relator groups are primitive recursive ..................

Clifton Earle Corzatt, Permutation polynomials over the rational numbers ...........

L. S. Dube, An inversion of the $S_{2}$ transform for generalized functions . . . . . . . . . . William Richard Emerson, Averaging strongly subadditive set functions in unimodular

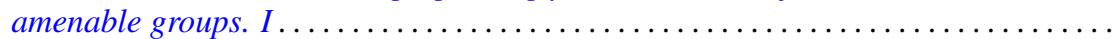

Barry J. Gardner, Semi-simple radical classes of algebras and attainability of

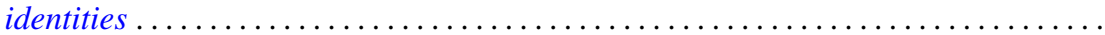

Irving Leonard Glicksberg, Removable discontinuities of A-holomorphic functions ....

Fred Halpern, Transfer theorems for topological structures . . . . . . . . . . . . . . .

H. B. Hamilton, T. E. Nordahl and Takayuki Tamura, Commutative cancellative

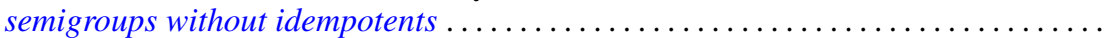

Melvin Hochster, An obstruction to lifting cyclic modules .....................

Alistair H. Lachlan, Theories with a finite number of models in an uncountable power

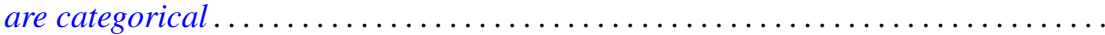

Kjeld Laursen, Continuity of linear maps from $C^{*}$-algebras . . . . . . . . . . . . .

Tsai Sheng Liu, Oscillation of even order differential equations with deviating arguments ....

Jorge Martinez, Doubling chains, singular elements and hyper- $Z$

Mehdi Radjabalipour and Heydar Radjavi, On the geometry of num Thomas I. Seidman, The solution of singular equations, I. Linear equations in Hilbert

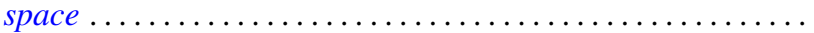

R. James Tomkins, Properties of martingale-like sequences ......

Alfons Van Daele, A Radon Nikodým theorem for weights on von Neumann

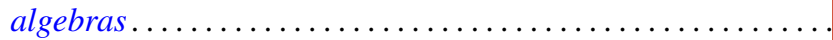

Kenneth S. Williams, On Euler's criterion for quintic nonresidues

Scott Andrew Wolpert, Noncompleteness of the Weil-Petersson metric for Teichmüller

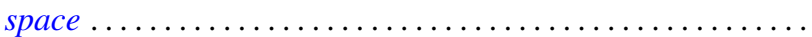

Volker Wrobel, Some generalizations of Schauder's theorem in locally convex

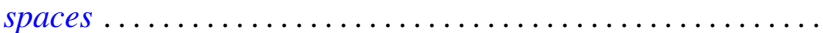

Kelly Denis McKennon, Corrections to: "Multipliers of type $(p, p)$ "; "Multipliers of type $(p, p)$ and multipliers of the group $L_{p}$-algebras"; "Multipliers and the

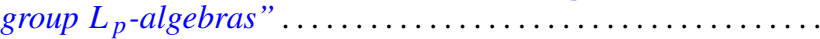

Andrew M. W. Glass, W. Charles (Wilbur) Holland Jr. and Stephen H. McCleary, Correction to: " $a *$-closures to completely distributive lattice-ordered

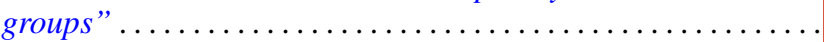

Zvi Arad and George Isaac Glauberman, Correction to: "A characteristic subgroup of

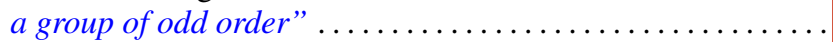

Roger W. Barnard and John Lawson Lewis, Correction to: "Subordination theorems

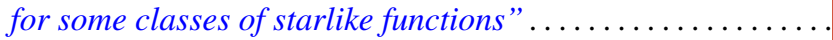

